\title{
Estrategia de gestión y divulgación del capital intelectual: influencia del gobierno corporativo
}

\author{
Management strategy and intellectual capital disclosure: influence of \\ corporate governance \\ Francisca Tejedo-Romero ${ }^{1, *}$ y Joaquim Filipe Ferraz Esteves Araujo ${ }^{2}$ \\ ${ }^{1}$ Universidad de Castilla-La Mancha, España \\ ${ }^{2}$ Universidade do Minho, Portugal
}

Recibido el 22 de septiembre del 2016; aceptado el 06 de junio del 2017

Disponible en Internet el 19 de marzo de 2018

\section{Resumen}

El conocimiento se ha convertido en un recurso estratégico clave para el éxito de los negocios y ha llevado a las empresas a preocuparse por la gestión de sus activos de conocimiento capital intelectual). No obstante, la contabilidad, como sistema de información, ha sufrido una pérdida de fiabilidad de la información suministrada, al poner en evidencia que el valor de mercado de las empresas es superior al valor contable que figura en los balances. Esta discrepancia ha sido uno de los principales motivos por los que las empresas gestionan y divulgan voluntariamente su capital intelectual. Apoyándonos en la teoría de la agencia y en la de recursos y capacidades, se analiza la influencia que ejerce el Gobierno Corporativo en la gestión de información del capital intelectual. Se utiliza como metodología el análisis de contenido de los informes anuales y se realiza un análisis multivariante de regresión lineal.

Códigos JEL: M19, M49

Palabras claves: Conocimiento, Capital intelectual, Gobierno Corporativo, Divulgación de Información.

\footnotetext{
*Autor para correspondencia

Correo electrónico: francisca.tejedo@uclm.es (F. Tejedo-Romero)

La revisión por pares es responsabilidad de la Universidad Nacional Autónoma de México.
} 


\begin{abstract}
Knowledge has become a key strategic resource for business success and has led companies to be concerned about managing their knowledge assets (intellectual capital). However, accounting as an information system has suffered a loss of reliability of the information provided, by showing that the market value of companies is higher than the book value shown in the balance sheets. This discrepancy has been one of the main reasons why companies voluntarily manage and disclose their intellectual capital. Relying on the agency theory and the resources and capabilities theory, this paper analyses the influence of corporate governance in the management of information intellectual capital. The methodology used was based on content analysis of annual reports using a analysis multivariate linear regression.
\end{abstract}

JEL Classification: M19, M49

Keywords: Knowledge, Intellectual Capital, Corporate Governance, Information Disclosure.

\title{
Introducción
}

Durante las últimas décadas se ha prestado especial atención al Gobierno Corporativo, en especial, tras los escándalos financieros protagonizados por algunas empresas, debido a las prácticas fraudulentas llevadas a cabo en la gestión de las mismas. Una de estas prácticas puede tener su origen en la escasa diligencia en la aplicación de las normas contables (Babío \& Muíño, 2005; Rodríguez-Ariza, Frías \& García, 2014; Sánchez Fernández de Valderrama, 2004), que ha generado una distorsión de la información financiera publicada, dando lugar a una desconfianza en el sistema económico actual. Además, estas actuaciones han contribuido a una crisis de valores y de actitudes básicas para mantener relaciones estables (Bueno Campos, 2002) entre propietarios y directivos, y otros grupos de interés de las empresas. La clave para subsanarlo está en los "Consejos de Administración" de las empresas que han actuado sin un adecuado Buen Gobierno y sin estándares éticos, ofreciendo, al mercado, una información distorsionada de la verdadera situación de las mismas y de su gestión (Briozzo, Albanese, \& Santolíquido, 2017; Hidalgo, García-Meca \& Martínez, 2011; Sánchez Fernández de Valderrama, 2004).

En este contexto, la falta de consenso teórico de principios generalmente aceptados o de modelos generales de medición, validados en su aplicación por la comunidad científica y profesional, ha provocado ciertas desviaciones interpretativas sobre la valoración de las empresas (Bueno Campos, 2002, p. 159; Tejedo-Romero \& Araujo, 2015), originando, en algunos casos, diferencias entre el valor de mercado y el que figura en las cuentas anuales. Algunos autores (Alcaniz, Gomez-Bezares \& Ugarte, 2015; Brooking, 1997; Edvinsson \& Malone, 1999) justifican dichas diferencias, entre otras cosas, al escaso reconocimiento del capital intelectual (en adelante CI) en la valoración de las empresas, a pesar de haberse convertido en un recurso económico clave (Hidalgo \& García-Meca, 2009; Spagnol, Moraes \& Piqueira, 2015; Villegas, Hernández \& Salazar, 2017; Zerenler, Hasiloglu \& Sezgin, 2008), cuyos orígenes se encuentran en: las personas (Capital Humano, en adelante $\mathrm{CH}$ ), el entorno socioeconómico (Capital Relacional, en adelante CR) y la organización (Capital Estructural, en adelante CE), cobrando un papel crítico en la creación de valor (de Cássia Fucci-Amato \& Amato-Neto, 2010; Herrera \& Macagnan, 2016; Villegas et al., 2017). Y, aunque el sistema de información contable juega un papel fundamental como generadora y suministradora de información, en la actualidad, no resulta de total utilidad para las empresas intensivas en CI- 
Conocimiento (An, Davey, Eggleton \& Wang, 2015; Brooking, 1997; Edvinsson \& Malone, 1999). El balance ha dejado de ser totalmente explicativos de la realidad empresarial sobre la que pretenden informar, ya que los criterios de identificación, medición y valoración han dejado de responder a las características de la economía basada en el conocimiento (Brennan, 2001; Herrera \& Macagnan, 2016; Hidalgo \& García-Meca, 2009; Tejedo-Romero \& Araujo, 2015).

Por esta razón, una serie de empresas e instituciones se han inclinado por la adopción de una perspectiva no estrictamente financiera en la valoración del CI, complementando los estados financieros anuales con información voluntaria. Esto requiere de una visibilidad más completa y transparente de la información divulgada por las empresas, al ser un elemento clave de un Buen Gobierno Corporativo. Así, no sería posible un buen gobierno corporativo sin un sistema contable eficiente, ni la calidad ofrecida por la contabilidad tendría fiabilidad sin un eficiente sistema de Gobierno Corporativo (Briano \& Saavedra, 2015; Briozzo et al., 2017; RodríguezAriza et al., 2014; Sánchez Fernández de Valderrama, 2004). Por ello, para la aplicación de prácticas de Buen Gobierno Corporativo, promoviendo eficiencia y transparencia, equidad en el tratamiento de los accionistas y difusión de información, así como el establecimiento de mecanismos efectivos de control interno, se ha producido una importante oleada de códigos de buen gobierno (CNMV, 1998, 2003, 2006, 2013 y 2015), para fomentar los comportamientos éticos en los órganos de administración y dirección de las empresas.

En este contexto, el objetivo de este trabajo se ha centrado en el estudio de la influencia de la estructura de propiedad y de determinadas características del Consejo de Administración de las empresas en una mayor transparencia informativa respecto al CI, tratando de aportar un mayor conocimiento, a los trabajos previos en este campo, sobre los cambios experimentados en la política divulgativa empresarial. La justificación para considerar como factor determinante al gobierno de la empresa, como señalan Li, Pike y Haniffa (2008), es que el consejo de administración promueve la divulgación de información en los informes anuales y, por tanto, las características de éste pueden ser importantes para influir en la política divulgativa de CI.

A este fin, y utilizando la metodología del "análisis de contenido", se han creado índices de divulgación, construyendo cuatro modelos estadísticos, con la técnica de regresión lineal, que permiten determinar la influencia que ejercen las variables representativas de la estructura de propiedad y de las características del Consejo de Administración de la empresa en la divulgación de información sobre el CI, en general y en particular, del CH, CR y CE. Además, se han considerado determinadas variables de control como tamaño, endeudamiento y sector. Para ello, y considerando la entrada en vigor de la ley 26/2003, de 17 de julio, de transparencia informativa, se seleccionaron los informes anuales de una muestra de 23 empresas cotizadas, incluidas en el índice bursátil del IBEX 35, durante los años 2004 a 2008, analizándose un total de 115 informes anuales, y dejando, para posteriores trabajos, el periodo 2009-2015, tras la aprobación de la ley 16/2007, 4 de julio, de reforma y adaptación de la legislación mercantil en materia contable para su armonización internacional con base en la normativa de la Unión Europea, y también al coincidir, a raíz de su entrada en vigor, con una serie de fusiones de empresas cotizadas en IBEX 35 a lo largo de los años 2009 y 2010, que hubiera distorsionado la serie histórica de las seleccionadas.

Asimismo, este estudio empírico ha requerido una importante búsqueda y recopilación de datos que, siendo de una gran especificidad, se encuentran dispersos en diversos informes, y que depende su divulgación, en gran medida, del Gobierno Corporativo (Consejo de Administración). Los resultados muestran que el porcentaje de acciones en manos de accionistas 
significativos, tamaño del consejo, separación de funciones (en el cargo de presidente y primer ejecutivo), porcentaje de consejeros independientes, tamaño, nivel de endeudamiento y sector de la empresa influyen en el nivel de divulgación de información sobre CI. Además, cabe destacar que la media de las empresas analizadas cumple con las recomendaciones establecidas en el código unificado de buen gobierno de 2006 (en adelante CUBG).

\section{Planteamiento de la investigación. Desarrollo de hipótesis EI Gobierno de la empresa}

Con la globalización de la economía y la liberalización de los mercados, como la desregulación, privatizaciones y venta de empresas públicas, se han incrementado los movimientos de capitales, produciéndose una mayor dispersión de la propiedad de las grandes empresas, debido a la existencia de una multitud de pequeños y grandes inversores (institucionales) que no participan en su gestión, pero que fueron adquiriendo el derecho a ejercer mayor protagonismo en la supervisión y control de las empresas, lo que lleva asociado el riesgo de conflictos de intereses (Berle \& Means, 1932) entre las partes. Este conflicto por la Teoría de la Agencia como un entramado de relaciones (relaciones de agencia) entre accionistas y directivos (Jensen \& Meckling, 1976), considerado eje central del problema del Gobierno de las empresas cotizadas. Esta teoría se fundamenta en que los directivos (agentes) pueden no actuar siempre en beneficio de los accionistas (principal), porque sus objetivos son diferentes. La resolución de este problema de agencia requiere el diseño de mecanismos de gobierno que permitan el control eficiente de la dirección de la empresa (Briano \& Saavedra, 2015; Briozzo et al., 2017; Hidalgo et al., 2011).

Por ello, con la finalidad de mejorar el Buen Gobierno de las empresas cotizadas, se han redactado Códigos de Buen Gobierno (CNMV, 1998, 2003, 2006, 2013 y 2015) donde se recogen una serie de recomendaciones y reglas dirigidas a los gobiernos de las mismas, para que voluntariamente las pongan en práctica (Babío \& Muíño, 2005), haciendo que los Consejos de Administración cumplan su función de mecanismo interno de supervisión y control (Rodríguez-Ariza et al., 2014).

En consecuencia, y sustentado en la ética y la transparencia, el Buen Gobierno es un medio eficaz para evitar los conflictos de intereses y los desequilibrios existentes entre accionistas mayoritarios y minoritarios, accionistas y directivos. Esto ha llevado a las empresas a mejorar e incrementar la transparencia mediante un mayor nivel de información divulgada, así como a una mayor accesibilidad y publicidad, tratando de devolver, a los mercados y a la sociedad en su conjunto, la confianza perdida.

\section{La información sobre capital intelectual}

Con el fin de solventar las limitaciones de la normativa contable vigente respecto a la identificación, medición y valoración del CI, y para mejorar la utilidad de la información que se proporciona a los accionistas, se ha sugerido que las empresas procedan a revelarla de manera voluntaria en secciones, bien dentro del informe anual, ya que es el principal medio de comunicación corporativa de las actividades e intenciones futuras de las empresas, o bien, por medio de los denominados informes de CI e informes de Sostenibilidad (An et al., 2015; Meritum, 2002; Rodrigues, Tejedo-Romero \& Craig, 2016). 
Así, la mayoría de los trabajos sobre divulgación de CI se han basado en el marco conceptual Sveiby (1997) para definir, clasificar y registrar la información sobre éste, siendo posteriormente desarrollado por Guthrie y Petty (2000), y aplicado en los estudios realizados por éstos en Australia, y replicado por: Brennan (2001) en Irlanda; Bozzolan et al. (2003) en Italia; April, Bosma y Deglon (2003) y Wagiciengo y Belal (2012) en Sudáfrica; Goh y Lim (2004) y Ahmed Haji y Mohd Ghazali (2013) en Malasia; An et al. (2015) en China; Abeysekera y Guthrie (2005) en Sri Lanka; Vandemaele et al. (2005) en los Países Bajos, Suecia y Reino Unido; Oliveras y Kasperskaya (2005) y Alcaniz et al. (2015) en España; Rodrigues, Tejedo-Romero \& Craig (2016) en Portugal; Hidalgo \& García-Meca (2009) e Hidalgo et al. (2011) en México; Herrera \& Macagnan (2016) en Brasil y España; De Silva, Stratford y Clark (2014) en Nueva Zelanda, entre otros. Estos trabajos demuestran que, a pesar de la inexistencia de una normativa regulatoria contable sobre el registro de los hechos de CI, las empresas están suministrando, de manera voluntaria, información relativa al mismo. En este sentido, nuestro trabajo se apoya en la teoría de recursos y capacidades puesto que, el CI es el recurso más importante que posee la empresa por su potencial estratégico en generar ventajas competitivas (Hall, 1992; Wagiciengo \& Belal, 2012). Muchas empresas están interesadas en informar de manera voluntaria con el fin de señalar su ventaja competitiva (Ahmed Haji \& Mohd Ghazali, 2013; Sonnier, 2008) y así, poder conseguir el apoyo de los inversores en el mercado de capitales (Alcaniz et al., 2015; Tabares, Alvarez \& Urbano, 2015).

\section{Desarrollo de hipótesis}

La presencia de diversos problemas en el Buen Gobierno de las empresas se puede deber, en algunos casos, a deficiencias en su política de presentación y divulgación de la información (Whittington, 1993), siendo la cantidad y calidad de ésta fundamental para reducir las asimetrías informativas y la discrecionalidad de los directivos, ya que limitan la libre actuación de la administración y dirección, e incentivan las prácticas manipuladoras (Babío \& Muiño, 2005; Prado, García \& Gallego-Álvarez, 2009; Rodríguez-Ariza et al., 2014; Rodrigues, TejedoRomero \& Craig, 2016). A este fin, y teniendo como fundamento la Teoría de la Agencia, se estudian la estructura de la propiedad y las características del Consejo de Administración, como factores influyentes en la divulgación de información del CI.

La estructura de la propiedad cobra especial importancia tanto por el grado de concentración o dispersión de la propiedad como por la participación accionarial de directivos y consejeros. Y se define como el grado de participación en la propiedad de la empresa que determina la distribución de poder y control (Briano \& Saavedra, 2015). Así, una alta dispersión de la propiedad de las acciones (gran número de pequeños accionistas) supone una mayor separación entre propiedad y gestión, originando unos elevados costos de agencia, debido a que éstos se encuentran alejados de la estructura de poder y declinan de su ejercicio y participación en la gestión y control de la empresa, derivando hacia la existencia de asimetrías informativas. Como señala Eng y Mak (2003), es necesaria una mayor supervisión y control por parte de los accionistas minoritarios, lo que requiere la necesidad de una mayor divulgación de información y, por ende, de CI. En virtud de este planteamiento se propone la primera hipótesis: 
H1: Existe una asociación negativa entre la concentración accionarial y el nivel de divulgación de información de CI.

Además, determinadas características del Consejo de Administración favorecen la articulación de reglas y conductas que pueden contribuir a mejorar la labor de supervisión y control, $y$, por ende, a un incremento de la transparencia informativa y de la confianza de los futuros inversores, minorando de esta manera el posible conflicto de intereses entre insiders (agente) y outsiders (principal).

La existencia de consejeros independientes puede favorecer la toma de decisiones orientadas a revelar información voluntaria (Babío \& Muíño, 2005; Barako, Hancock, \& Izan, 2006; Briano \& Saavedra, 2015; Cerbioni \& Parbonetti, 2007; Chen \& Jaggi, 2000; Gisbert \& Navallas, 2013; Hidalgo et al., 2011; Ho \& Wong, 2001; Li et al., 2008; Lim, Matolcsy, \& Chow, 2007) de CI en los informes anuales, reduciendo las asimetrías de información entre los directivos y accionistas (Hidalgo et al., 2011; Lim et al., 2007). Puesto que se espera más objetividad e independencia en el análisis de la gestión y del comportamiento de la empresa por parte de los independientes (Rodríguez-Ariza et al., 2014). Por tanto, esta característica del Consejo suele mejorar su capacidad decisoria de supervisión y control para aliviar los conflictos de agencia entre propietarios-accionistas y directivos, e incrementar el nivel de divulgación de información de CI. Por ello, se plantea la siguiente hipótesis:

H2: Existe una asociación positiva entre la independencia del Consejo de Administración y el nivel de información divulgada de CI.

Cuando el cargo de presidente del Consejo y primer ejecutivo recae en la misma persona, puede dar lugar a comportamientos ineficaces y oportunistas (Jensen \& Meckling, 1976), debido a la excesiva concentración de poder, aunque, teóricamente, debería favorecer la divulgación de información para disminuir los costos de coordinación (Coles, Daniel, \& Naveen, 2008; Hidalgo et al., 2011; Jensen, 1993). Li et al. (2008) sostienen que la dualidad en el cargo puede limitar la independencia del Consejo, y poner en peligro las funciones de control y supervisión que afectan a la política divulgativa de información de la empresa. La concentración de mucho poder en manos de una única persona puede dar lugar a comportamientos ineficaces y oportunistas (Jensen \& Meckling, 1976), favoreciendo los intereses personales en detrimento de la empresa (Prado et al., 2009). La tercera hipótesis a corroborar será:

H3: La divulgación de información de CI está positivamente relacionada con la separación de las funciones de primer ejecutivo y presidente del consejo.

En la literatura previa no se detecta una posición unánime en relación con el tamaño del Consejo de Administración a la hora de garantizar la eficiencia en su rol de supervisión y control (Rodrigues, Tejedo-Romero \& Craig, 2016). Algunos autores, como Pearce y Zahra (1992), opinan que un tamaño del Consejo elevado favorece la diversidad de criterio y el nivel de información divulgada. En esta línea, Briano y Saavedra (2015), Gisbert y Navallas, (2013) e Hidalgo et al. (2011) encuentran una asociación positiva entre el tamaño del Consejo y el nivel de divulgación voluntaria de información. Sin embargo, Jensen (1993) considera que ello se torna en reducción de la rapidez y eficiencia en la toma de decisiones por falta de coordinación e información. Aunque un elevado número de miembros en el Consejo puede suponer una mayor capacidad de supervisión, ésta puede mermarse por un alargamiento en el proceso de toma de decisiones y en el procedimiento de comunicación (Jensen, 1993), y, por tanto, estaría negativamente relacionados con la transparencia informativa de CI. En este sentido, Cerbioni y Parbonetti (2007) y Lim et al. (2007) encuentran una asociación negativa entre el tamaño del 
Consejo y el nivel de información voluntaria. Por tanto, se plantea la siguiente hipótesis:

H4: La divulgación de información de CI está negativamente relacionada con el tamaño del Consejo de Administración.

\section{Diseño metodológico y obtención de datos}

Para este estudio empírico (contrastar las hipótesis), se ha empleado la metodología del análisis de contenido (April et al., 2003; Beattie \& Thomson, 2007; Bozzolan et al., 2003; Guthrie, Petty, Yongvanich, \& Ricceri, 2004; Tejedo-Romero \& Araujo, 2015), cuyas técnicas han servido como base para la elaboración de un índice de divulgación y tres subíndices (Rodrigues, Tejedo-Romero \& Craig, 2016) con la finalidad de poder cuantificar la información sobre el CI, en general y en particular, del CH, CR y CE de las empresas objeto de estudio.

Para garantizar la fiabilidad del análisis de contenido se han considerado las indicaciones de Guthrie et al. (2004) que consideran que, para el caso de un único codificador si éste ha sido objeto de un período suficiente de formación y las decisiones de codificación han llegado a un nivel aceptable en la muestra piloto realizada, se puede probar la fiabilidad en el proceso de depuración de los datos. No obstante, para suplir la falta de transparencia en la codificación de los mismos (Beattie \& Thomson, 2007), se han llevado a cabo las etapas que se desarrollan a continuación.

\section{Periodo de estudio}

El periodo elegido abarca 5 años, desde el 2004 al 2008 (ambos inclusive). Esta elección viene motivada, como ya se expuso, por la aprobación en España de la ley 26/2003, de 17 de julio, de transparencia informativa, que regula a todas las sociedades emisoras de valores e instrumentos financieros admitidos a cotización, donde se recoge, en el apartado 2 del artículo 117 , que dichas sociedades deben disponer de una página web para difundir información, lo que facilita el acceso, a partir de 2004, a los documentos analizados de forma más fácil y accesible.

\section{Definición de la población y elección de la muestra}

Las empresas cotizadas en el mercado continuo español que estuviesen incluidas en el índice bursátil del IBEX 35 durante el período 2004-2008 constituyen la población de partida, al ser éstas más susceptibles de una mayor transparencia informativa (Hernández, Aibar, \& Aibar, 2015). El IBEX 35 es el principal índice bursátil de referencia de la bolsa española. Está formado por las 35 empresas con más liquidez que cotizan en la bolsa; son las que poseen un mayor volumen de contratación y capitalización bursátil.

En el proceso de selección de la muestra se ha optado por un diseño dirigido o no probabilístico (Hernández, Fernández, \& Baptista, 2006), partiendo de las empresas incluidas en el índice del 2008 y manteniéndolas fijas durante el resto de años analizados, hasta el año 2004. Se optó por el año 2008 con la finalidad de obtener una muestra significativa de las empresas del IBEX 35, puesto que, en años posteriores, han tenido lugar operaciones de fusiones, y algunas empresas han salido de dicho índice. Así, la muestra quedó configurada por 23 grupos empresariales, disponiendo de información a nivel de grupo, consolidada, un 65,7\% de la población de partida. Igualmente, se encuentran caracterizados todos los sectores industriales (ver Figura 1). 
Figura 1. Número de empresas por sectores en la muestra y en la población.

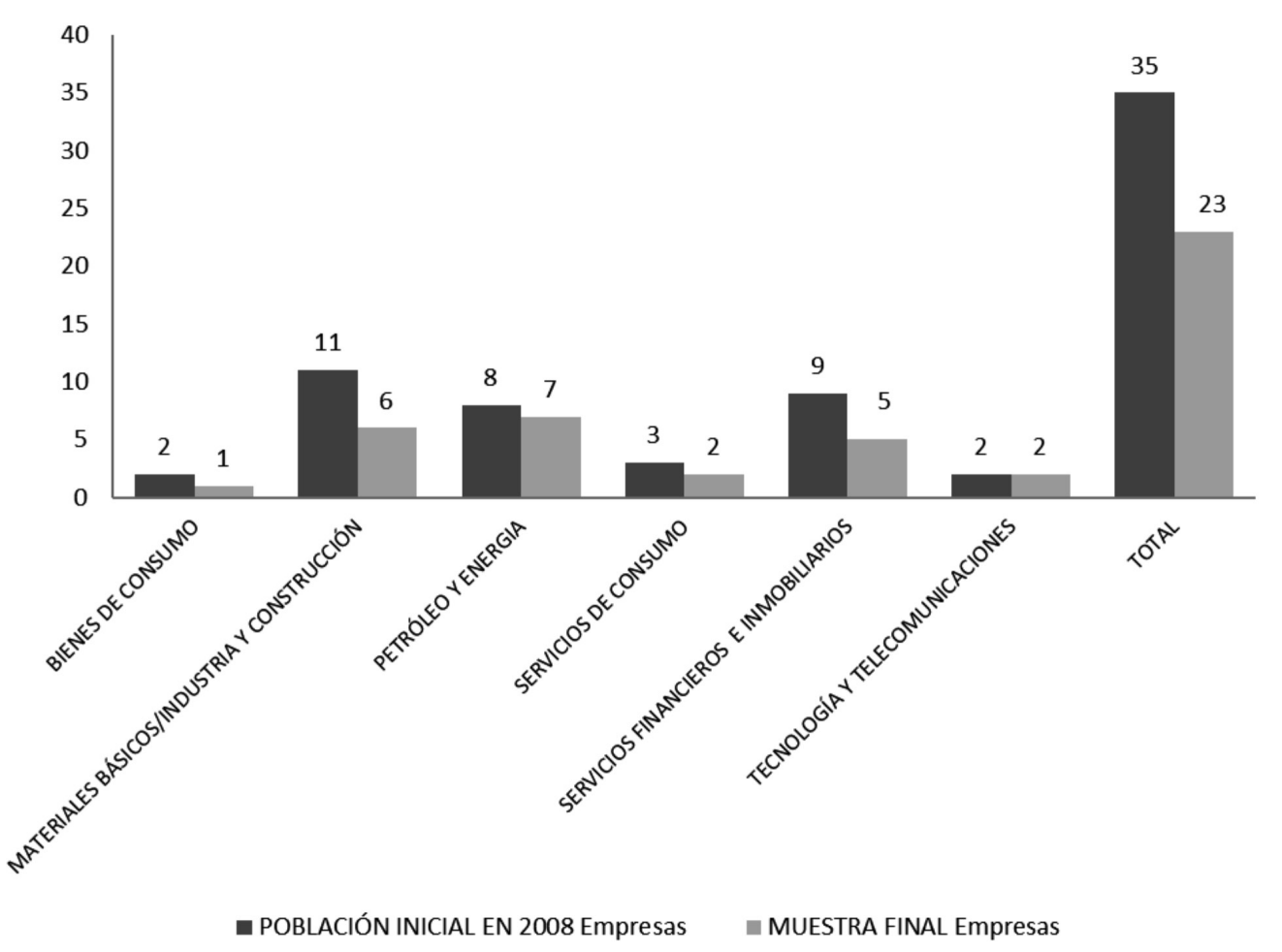

\section{Medición de las variables y obtención de los datos}

Con la finalidad de corroborar las hipótesis que se han planteado previamente, el nivel de divulgación de información sobre el CI, en general y en particular, CH, CR y CE, suministrado por las empresas en sus informes o memorias anuales, han sido las variables dependientes que se han considerado para los cuatro modelos estadísticos que se especifican más adelante. Estas variables se han medido utilizando índices y subíndices de divulgación no ponderados que se basan en los resultados obtenidos del análisis de contenidos de los informes anuales. Así, las unidades de mиestreo han sido los informes anuales, las de registro son la presencia o ausencia de información de CI y las unidades contexto se ha analizado la información a nivel de frase. Para la codificación de los datos se ha seguido el marco inicial de Sveiby (1997). Así, se utiliza un modelo tripartito de $\mathrm{CI}$ donde cada categoría ( $\mathrm{CH}, \mathrm{CR}$ y $\mathrm{CE}$ ) se compone de una serie de subcategorías y de elementos específicos que se han basado en la literatura previa (ver tabla 1). 
Tabla 1

Categorías, subcategorías y elementos intangibles

\begin{tabular}{|c|c|c|}
\hline CH & CR & CE \\
\hline EMPLEADOS: & 21. RELACIONES FINANCIERAS & PROPIEDAD INTELECTUAL: \\
\hline 1. Perfil del empleado & 22. $\underline{M A R C A S}$ & 42. Patentes \\
\hline 2. Datos económicos & CLIENTES: & 43. Copyrights \\
\hline 3. Seguridad y salud de los empleados & 23. Nombre de clientes relevantes & 44. Marcas comerciales \\
\hline 4. Relaciones laborales y actividad sindical & 24. Lealtad de clientes & 45. Secretos comerciales \\
\hline 5. Implicación de los trabajadores con la & 25. Satisfacción del cliente & 46. FILOSOFÍA DE GESTIÓN \\
\hline comunidad & 26. Servicios y apoyo a clientes & 47. CULTURA CORPORATIVA \\
\hline 6. Reconocimiento de los empleados & REPUTACIÓN/IMAGEN DE LA & PROCESOS TECNOLÓGICOS Y DE GESTIÓN: \\
\hline 7. Empleados destacados & EMPRESA: & 48. Procesos de Gestión \\
\hline 8. Temas de igualdad de oportunidades y & 27. Nombre de la empresa & 49. Procesos Tecnológicos \\
\hline diversidad & 28. Contratos favorables & $\underline{I+D+i}:$ \\
\hline 9. Comportamiento de empleados & 29. Medidas de protección del & $\overline{50 . \text { Investigación y Desarrollo }}$ \\
\hline 10. Compromisos de empleados & medioambiente & 51. Innovación \\
\hline 11. Motivación de los empleados & 30. Patrocinio y mecenazgo & SISTEMAS DE INFORMACIÓN Y DE REDES: \\
\hline EDUCACIÓN: & 31. Responsabilidad social/Acción social & 52. Sistemas de información \\
\hline 12. Educación reglada & 32. Gobierno de la empresa & 53. Sistemas de redes \\
\hline 13. Cualificación profesional & SOCIOS DEL NEGOCIO: & \\
\hline FORMACIÓN Y DESARROLLO: & 33. Colaboración con empresas & \\
\hline 14. Formación de empleados & 34. Acuerdos de franquicia & \\
\hline 15. Desarrollo de empleados & 35. Acuerdos de licencia & \\
\hline CONOCIMIENTOS RELACIONADOS CON & OTRAS RELACIONES DE LA EMPRESA & \\
\hline EL TRABAJO: & 36. Relaciones con proveedores & \\
\hline 16. Know-how (saber hacer) & 37. Relaciones con las administraciones & \\
\hline 17. Experiencia profesional & públicas & \\
\hline 18. Antigüedad de los expertos) & 38. Relaciones con centros de & \\
\hline 19. Rendimiento y resultado de los altos & investigación & \\
\hline directivos & 39. Relaciones con los medios de & \\
\hline \multirow{3}{*}{ 20. ESPÍRITU EMPRENDEDOR } & comunicación & \\
\hline & 40. Relaciones con otros grupos de interés & \\
\hline & 41. CANALES DE DISTRIBUCIÓN & \\
\hline
\end{tabular}

Partiendo del marco anterior, del índice general de CI (ICI), se han elaborado tres subíndices correspondientes a las categorías de $\mathrm{CH}$ (ICH), CR (ICR) y CE (ICE), configurándose cada uno de éstos de la siguiente manera:

$$
I C I_{j}=\frac{1}{53} \sum_{i=1}^{53} X_{i j} ; I C H_{j}=\frac{1}{20} \sum_{i=1}^{20} X_{i j} ; I C R_{j}=\frac{1}{21} \sum_{i=21}^{41} X_{i j} ; I C E_{j}=\frac{1}{12} \sum_{i=42}^{53} X_{i j}
$$

Donde $I_{j}$ son el índice y subíndices absoluto de divulgación no ponderado de la empresa $j, i$ son los ítems o partidas, $j$ es la empresa y, $X_{i j}$ es la puntuación obtenida para el ítem $i$ en la empresa $j$. De modo que, $X i j$ tomará el valor 1 si la empresa $j$ ha divulgado el ítem $i$ y, tomará el valor 0 en caso contrario.

Con relación a las variables independientes, los datos necesarios se han obtenido de los informes anuales de Gobierno Corporativo. La medición de estas variables queda descrita a continuación: 
Tabla 2

Variables independientes

\begin{tabular}{lll}
\hline VARIABLE & MEDIDA \\
\cline { 2 - 3 } $\begin{array}{l}\text { Concentración } \\
\text { accionarial }\end{array}$ & $\begin{array}{l}\text { Variable numérica que representa la tenencia de acciones por persona física o } \\
\text { jurídica } \geq 5 \% \text { del capital. }\end{array}$ \\
$\begin{array}{l}\text { Independencia } \\
\text { del Consejo de } \\
\text { Administración }\end{array}$ & $\begin{array}{l}\text { Variable numérica que representa el porcentaje de consejeros independientes en el } \\
\text { Consejo de Administración. }\end{array}$ \\
$\begin{array}{l}\text { Separación de } \\
\text { las funciones de } \\
\begin{array}{l}\text { Primer Ejecutivo } \\
\text { y Presidente del } \\
\text { Consejo }\end{array}\end{array}$ & $\begin{array}{l}\text { Variable dummy que toma el valor de } 1 \text { en caso de separación de funciones y } 0 \text { en } \\
\text { caso contrario. }\end{array}$ \\
$\begin{array}{l}\text { Tamaño del } \\
\text { Consejo de }\end{array}$ & - & $\begin{array}{l}\text { Variable numérica que representa el número total de miembros que integran el } \\
\text { Administración }\end{array}$ \\
\hline
\end{tabular}

Además, se han considerado determinadas características empresariales como variables de control (Alcaniz et al., 2015; Chen \& Jaggi, 2000; Rodrigues, Tejedo-Romero \& Craig, 2016), puesto que son necesarias para aislar el efecto de las características del Consejo de administración en el nivel de divulgación de información sobre CI. La obtención de los datos se ha realizado desde la base de datos SABI (Sistema de Análisis de Balances Ibéricos).

Tamaño: Ha sido medida como el logaritmo neperiano del número de empleados (Alcaniz et al., 2015; Bozzolan et al., 2003).

Sector: Se han considerado variables dicotómicas para cada uno de los sectores, es decir recibe un valor igual a 1 si la empresa pertenece al sector en cuestión y un valor 0 si no pertenece (Hidalgo \& García-Meca, 2009; Rodrigues, Tejedo-Romero \& Craig, 2016).

Endeudamiento: Es medido como el cociente entre el importe total de la deuda y el patrimonio neto (Cerbioni \& Parbonetti, 2007). Además, se ha incluido el $\underline{a \tilde{n} o}$ como variable de control.

\section{Modelo de investigación}

De acuerdo con lo expuesto, para obtener las variables que, en mayor medida, contribuyen a explicar la variabilidad del índice (CI) y subíndices (CH, CR y CE) de información, se han utilizado cuatro modelos estadísticos de regresión lineal múltiple que permiten, dada una variable dependiente a explicar y un conjunto de variables independientes, obtener una función lineal de tales variables con la cual explicar o predecir el valor de la variable dependiente. A este fin, se crean los cuatro modelos estadísticos siguientes:

Modelo 1:

$$
I C I_{i t}=\alpha+\sum_{j=1}^{4} \beta_{j} \text { Variables de Gobierno }_{j i t}+\sum_{k=1}^{4} \gamma_{k} \text { Variables de Control }_{k i t}+\varepsilon
$$


Modelo 2:

$$
\text { ICH }_{i t}=\alpha+\sum_{j=1}^{4} \beta_{j} \text { Variables de Gobierno }_{j i t}+\sum_{k=1}^{4} \gamma_{k} \text { Variables de Control }_{k i t}+\varepsilon
$$

Modelo 3:

ICR $_{i t}=\alpha+\sum_{j=1}^{4} \beta_{j}$ Variables de Gobierno $_{j i t}+\sum_{k=1}^{4} \gamma_{k}$ Variables de Control Kit $+\varepsilon$

Modelo 4:

$I_{\text {ICE }}=\alpha+\sum_{j=1}^{4} \beta_{j}$ Variables de Gobierno $_{j i t}+\sum_{k=1}^{4} \gamma_{k}$ Variables de Control $_{k i t}+\varepsilon$

Donde:

ICI, ICH, ICR e ICE y = Índice de CI, CH, CR y CE.

Variables de Gobierno = relativas tanto a la estructura de la propiedad como a las características del Consejo de Administración.

Variables de control $=$ relativas a las características empresariales.

$B$ y $\gamma \gamma=$ Parámetros.

$\varepsilon=$ Término de error.

\section{Resultados y discusión}

En primer lugar, se llevó a cabo un análisis para efectuar una aproximación al comportamiento de las variables de los modelos mediante sus principales estadísticos.

Tabla 3

Estadísticos descriptivos de las variables dependientes e independientes del gobierno corporativo

\begin{tabular}{|c|c|c|c|c|c|c|}
\hline & $\mathbf{N}$ & Mínimo & Máximo & Media & Desv. típ. & Mediana \\
\hline \multicolumn{7}{|l|}{ Variables dependientes } \\
\hline ICI & 115 & 0 & 0.698 & 0.417 & 0.198 & 0.491 \\
\hline ICH & 115 & 0 & 0.800 & 0.386 & 0.219 & 0.450 \\
\hline ICR & 115 & 0 & 0.762 & 0.407 & 0.203 & 0.476 \\
\hline ICE & 115 & 0 & 0.917 & 0.487 & 0.234 & 0.583 \\
\hline \multicolumn{7}{|l|}{ Variables independientes } \\
\hline$\%$ Accionistas Significativos & 115 & $0 \%$ & $92.06 \%$ & $30.92 \%$ & $23.88 \%$ & $27.46 \%$ \\
\hline $\begin{array}{l}\text { \%Consejeros Independientes } \\
\text { en el CA }\end{array}$ & 115 & $9.09 \%$ & $80 \%$ & $40.66 \%$ & $17.03 \%$ & $41.18 \%$ \\
\hline \multirow[t]{2}{*}{ Tamaño Del Consejo } & 115 & 8 & 24 & 14.99 & 3.84 & 15 \\
\hline & $\mathbf{N}$ & Frecuencia & Porcentaje & $\begin{array}{l}\text { Porcentaje } \\
\text { acumulado }\end{array}$ & Media & Desv. típ. \\
\hline Separación Funciones & No Separación & 86 & 74.8 & 74.8 & 0.252 & 0.436 \\
\hline
\end{tabular}


Los resultados demuestran, que pese a estar inmersos en la Sociedad del Conocimiento, se divulga escasa información de CI, con una media en el nivel de información del CH, CR y CE de un 39\%, $41 \%$ y $49 \%$, respectivamente. Resultados muy similares a los obtenidos en trabajos previos de otros países: en Australia (Guthrie \& Petty, 2000), Irlanda (Brennan, 2001), Sudáfrica (April et al., 2003), Suecia, Países Bajos y Reino Unido (Vandemaele et al., 2005), un 30\%, 40\% y 30\%, respectivamente; en Italia (Bozzolan et al., 2003) un 21\%, 49\% y 30\%, respectivamente; en Sri Lanka (Abeysekera \& Guthrie, 2005) un 36\%, 44\% y 20\%, respectivamente; en Malasia (Goh \& Lim, 2004) un 22\%, $41 \%$ y 37\%, respectivamente; y en España (Oliveras \& Kasperskaya, 2005) un 21\%, 51\% y 28\%, respectivamente. Así, bajo los postulados de la teoría de los recursos y capacidades, las empresas tienen miedo a perder su ventaja competitiva que tiene su origen en el recurso estratégico más importante que poseen, su CI, y quizás puede ser el motivo de la escasa divulgación.

Finalmente, la estimación del modelo estadístico de regresión lineal se realiza mediante el método de regresión por etapas "hacia adelante", en el que la entrada y salida de las variables independientes en la ecuación de regresión se realiza a partir de los valores del estadístico $t$-Student. En cada paso se calcula el coeficiente estandarizado para cada variable si la misma entrase en el modelo en el próximo paso, así como el valor del estadístico $t$ y el nivel de significación. En este trabajo, se ha utilizado como criterio de entrada en la ecuación un nivel de significación inferior al 0,05 y, uno de salida, superior al 0,1. En este sentido, se exponen los resultados (ver tabla 4) que se han obtenido en los cuatro modelos estadísticos tras haberse cumplido los supuestos de linealidad, independencia, homocedasticidad, normalidad y no multicolinealidad para su correcta aplicación.

Tabla 4

Resultados de los modelos de regresión lineal

\begin{tabular}{|c|c|c|c|c|c|c|c|c|}
\hline \multirow[t]{2}{*}{ Variables } & \multicolumn{2}{|c|}{$\begin{array}{c}\text { Modelo } 1 \\
\text { CI }\end{array}$} & \multicolumn{2}{|c|}{$\begin{array}{c}\text { Modelo } 2 \\
\text { CH }\end{array}$} & \multicolumn{2}{|c|}{$\begin{array}{c}\text { Modelo } 3 \\
\text { CR }\end{array}$} & \multicolumn{2}{|r|}{$\begin{array}{c}\text { Modelo } 4 \\
\text { CE }\end{array}$} \\
\hline & $\beta$ & $\mathbf{t}$ & $\beta$ & 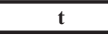 & $\beta$ & $\mathbf{t}$ & $\beta$ & $\mathbf{t}$ \\
\hline (Constante) & & $9.975 * * *$ & & $1.868^{*}$ & & $7.715^{* * *}$ & & $2.000^{* *}$ \\
\hline$\%$ Acc de actas significativos & & & & & 0.227 & $2.509^{* *}$ & & \\
\hline$\%$ Consejeros independientes & & & -0.225 & $-2.675^{* * *}$ & & & & \\
\hline Separación funciones & 0.365 & $3.983^{* * *}$ & & & 0.417 & $4.584 * * *$ & 0.281 & $3.070 * * *$ \\
\hline Tamaño del CA & -0.475 & $-4.989 * * *$ & -0.361 & $-4.352 * * *$ & -0.378 & $-4.144 * * *$ & -0.314 & $-3.444 * * *$ \\
\hline $\mathrm{N}^{\circ}$ empleados & 0.454 & $5.190 * * *$ & & & 0.573 & $6.148^{* * *}$ & 0.367 & $4.073 * * *$ \\
\hline Endeudamiento \% & & & .593 & $5.674^{* * *}$ & & & 0.392 & $3.450^{* * *}$ \\
\hline Servicios de consumo & -0.257 & $-3.199 * * *$ & -0.397 & $-5.070^{* * *}$ & -0.236 & $-2.915^{* * *}$ & -0.216 & $-2.683^{* * *}$ \\
\hline Bienes de consumo & -0.208 & $-2.484 * *$ & & & & & & \\
\hline Servicios financieros e inmobiliarios & & & -0.481 & $-4.571 * * *$ & & & -0.424 & -3.778 \\
\hline$R^{2}$ & & 329 & & .390 & & 40 & & 0.355 \\
\hline$R^{2}$ corregido & & 298 & & .362 & & 10 & & 0.320 \\
\hline$F$ & & $89 * * *$ & & $943 * * *$ & & $7 * * *$ & & $9.922 * * *$ \\
\hline
\end{tabular}

$* * *=$ Significativa para $\mathrm{p}<0,01 ; * *=$ Significativa para $\mathrm{p}<0,05 ; *=$ Significativa para $\mathrm{p}<0,1$ 
En cuanto al suministro de información de CI, se observa que son cuatro las variables que contribuyen a explicar los niveles de información: 1) El tamaño del Consejo resulta determinante en el nivel de información del CI $(\beta=-0,475$, sig. $=0,000)$, a mayor tamaño menos información se divulga, así se reconoce tanto a nivel teórico ( $\mathrm{T}^{\mathrm{a}}$ de la Agencia) como empírico (Babío \& Muíño, 2005; Cerbioni \& Parbonetti, 2007; Lim et al., 2007), corroborándose la cuarta hipótesis planteada. 2) La separación de funciones, en el cargo de presidente del Consejo y primer ejecutivo, es estadísticamente significativa $(\beta=0,365$, sig. $=0,000)$, implica un mayor nivel de divulgación de información sobre CI (Barako \& Brown, 2008; Cerbioni \& Parbonetti, 2007; Li et al., 2008), confirmándose de esta manera la hipótesis tercera. 3) El tamaño de la empresa resulta determinante $(\beta=0,454$, sig. $=0,000)$ para justificar mayores niveles de información divulgada de CI (Barako et al., 2006; Bozzolan et al., 2003; Haniffa \& Cooke, 2005; Ho \& Wong, 2001). Y, a nivel sectorial, se observa que el de servicios $y$ bienes de consumo mantienen comportamientos diferenciales $(\beta=-0,257$, sig. $=0,002 ; \beta=-0,208$, sig. $=0,015$, respectivamente) con respecto del resto de sectores, siendo éstos los que menos divulgación de información de CI realizan, puesto que son sectores poco intensivos en este tipo de capital (OECD, 2001).

El ajuste del modelo estadístico de CI medido por el $R^{2}$ corregido asciende a 0,298 , lo que implica que las variables independientes explican un $29,8 \%$ de la varianza del nivel de divulgación de la información del CI. Este valor es muy similar al obtenido en los estudios de Chen y Jaggi (2000) con un 30\%, Ho y Wong (2001) con un 31,4\%, Lim et al. (2007) con un $18,79 \%$.

En cuanto al suministro de información de $\mathbf{C H}$, las variables que contribuyen a explicar los niveles de información han sido: el tamaño del Consejo que resulta determinante en el nivel de información, a mayor tamaño menos información se divulga $(\beta=-0,361$, sig. $=0,000)$. Además, como ocurre en otros trabajos previos (Eng \& Mak, 2003; Haniffa \& Cooke, 2005), un mayor \% de consejeros independientes en el Consejo conlleva un menor nivel de información suministrado $(\beta=-0,225$, sig.=0,009), ya que como argumentan Li et al. (2008), puede ser que los consejeros no ejecutivos no sean necesariamente tan independientes. También, resulta significativo el nivel de endeudamiento $(\beta=0,593$, sig $=0,000)$ y los sectores de servicios de consumo y financiero $e$ inmobiliario $(\beta=-0,397$, sig. $=0,000 ; \beta=-0,481$, sig. $=0,000$, respectivamente). En este modelo, el $R^{2}$ corregido asciende a 0,362 , lo que implica que las variables independientes explican un $36,2 \%$ de la varianza del nivel de divulgación de la información del $\mathrm{CH}$, corroborándose de manera particular las hipótesis $\mathrm{H} 2$ y $\mathrm{H} 4$.

En el caso del suministro de información de $\mathbf{C R}$, e igual que para el caso del modelo 1 relativo a la información de CI, las variables que resultan significativas para explicar los niveles de información han sido: el tamaño del Consejo, la separación de funciones, el tamaño de la empresa y el sector de servicios de consumo $(\beta=-0,378$, sig. $=0,000 ; \beta=0,417$, sig $=0,000$; $\beta=0,573$, sig. $=0,000 ; \beta=-0,236$, sig. $=0,004$, respectivamente). También ha resultado significativa la variable \% de acciones en manos de accionistas significativos $(\beta=0,227$, sig. $=0,014)$, implicando un mayor nivel de información suministrada (Eng \& Mak, 2003). En cuanto al ajuste, este modelo estadístico presenta un $R^{2}$ corregido que asciende a 0,310 , lo que implica que las variables independientes explican un $31 \%$ de la varianza del nivel de divulgación de la información del CR. Así, se confirman las hipótesis H1, H3 y H4.

Las variables que contribuyen a explicar el suministro de información de $\mathbf{C E}$ son: el tamaño del Consejo, separación de funciones, tamaño de la empresa y el sector servicios de consumo 
$(\beta=-0,314$, sig. $=0,001 ; \beta=0,281$, sig. $=0,003 ; \beta=0,367$, sig. $=0,000 ; \beta=-0,216$, sig. $=0,008$, respectivamente), igual que ocurría en el caso de la información relativa al CI y CR. Asimismo, y al igual que para el caso del $\mathrm{CH}$, han resultado estadísticamente significativas el nivel de endeudamiento $(\beta=0,392$, sig. $=0,001)$, con una relación positiva (Barako et al., 2006; Eng \& Mak, 2003), y el sector financiero e inmobiliario $(\beta=-0,424$, sig. $=0,000)$, que es poco intensivo en intangibles (OECD, 2001). El $R^{2}$ corregido asciende a 0,320 , lo que implica que las variables independientes explican un 32\% de la varianza del nivel de divulgación de la información del CE, corroborándose las siguientes hipótesis: $\mathrm{H} 3$ y H4.

\section{Conclusiones}

Este estudio empírico evidencia cómo la existencia de determinadas características de Gobierno Corporativo, relativas a la estructura de la propiedad y al Consejo de Administración, influyen en la divulgación de información voluntaria respecto al CI. Los resultados avalan que la existencia de determinadas recomendaciones establecidas en España en el CUBG (CNMV, $2006,2013,2015)$ proporcionan los mecanismos de control necesarios para incrementar el grado de transparencia informativa, en aras a la existencia de un buen gobierno corporativo como medio de evitar los posibles conflictos de intereses desde la óptica de la Teoría de la Agencia.

Por tanto, el Gobierno Corporativo juega un papel fundamental a la hora de divulgar información voluntaria del CI, como mecanismo para reducir las asimetrías de información y los costes de agencia. En concreto, se ha evidenciado que, el porcentaje de acciones en manos de accionistas significativos, el tamaño del Consejo de Administración, la separación de funciones (en el cargo de presidente y primer ejecutivo), el porcentaje de consejeros independientes miembros del Consejo de Administración influyen en el nivel de divulgación de información. Asimismo, se ha evidenciado como determinadas características empresariales como el tamaño, sector y endeudamiento influyen en el suministro de una mayor cantidad de información voluntaria.

En este sentido, se ha confirmado que un mayor número de miembros pertenecientes al Consejo perjudica los niveles de divulgación de información del CI, CH, CR y CE suministrados por la empresa; es decir un mayor tamaño del Consejo tiene un impacto negativo sobre la efectividad del Consejo debido a que los consejeros podrían estar menos motivados para participar en la toma de decisiones estratégicas y revelarían menos información. Además, la separación de funciones es un mecanismo útil para salvaguardar los interesas de todas las partes, mejorando la función de supervisión y la cantidad de información divulgada relativa al CI, CR y CE. Asimismo, un mayor número de consejeros independientes influyen negativamente en el suministro de información de $\mathrm{CH}$; quizás pueda deberse a que estos consejeros no son tan independientes como en un principio se pensaba, o bien, porque la presencia de ellos dentro del Consejo de Administración (que garantiza los intereses de los accionistas minoritarios y del resto de stakeholders) sea el motivo de que los accionistas minoritarios demanden menos información, al tener depositado en los independientes una mayor confianza, y la empresa divulgue menos. Finalmente, alto grado de concentración de la propiedad en manos de los principales accionistas beneficia la divulgación de información sobre el CR; esto puede deberse a que estén interesados en revelar información del CR con la finalidad de aumentar la liquidez 
y el valor de las acciones de la empresa.

Bajo el marco de la Teoría de la Agencia, con estos resultados, se ha pretendido contribuir al estudio de los mecanismos de Gobierno Corporativo y su influencia en la divulgación de información del CI, aportando luz de la situación en España, debido a los escasos trabajos previos. Además, esta aportación es de una gran utilidad estratégica para reducir el problema de agencia, que puede ir encaminada para futuras recomendaciones y modificaciones de los códigos existentes, siendo de interés para reguladores, analistas financieros, inversores, participantes en el mercado de capitales, etc.

En este sentido, nuestro trabajo ha tratado de aportar evidencia de lo recomendable de impulsar el Buen Gobierno Corporativo, los códigos de conducta y comportamientos éticos en los órganos de administración y gestión de las empresas, con la finalidad de incrementar la transparencia informativa. Además, también se ha pretendido evidenciar la utilidad que, para el resto de empresas españolas, supone conocer la actuación de la alta dirección e importancia dada a la divulgación voluntaria sobre CI y los factores determinantes de ese mayor nivel de información.

Por último, significar que un trabajo futuro irá encaminado a completar la metodología del análisis de contenido con una triangulación metodológica, elaborando un cuestionario dirigido a los encargados de elaborar los informes o memorias anuales. Además, nos gustaría ampliar la muestra objeto de estudio en un contexto internacional, estudiando las posibles diferencias existentes en relación con los mecanismos de gobierno corporativo en cada uno de los países analizados; así como, ampliar el periodo objeto de estudio incorporando datos más actuales correspondiente al periodo 2009-2017.

\section{Referencias}

Abeysekera, I., \& Guthrie, J. (2005). An empirical investigation of annual reporting trends of intellectual capital in Sri Lanka. Critical Perspectives on Accounting, 16(3), 151-163. http://dx.doi.org/10.1016/s1045-2354(03)00059-5.

Ahmed Haji, A., \& Mohd Ghazali, N. A. (2013). A longitudinal examination of intellectual capital disclosures and corporate governance attributes in Malaysia. Asian Review of Accounting, 21(1), 27-52. https://doi. org/10.1108/13217341311316931

Alcaniz, L., Gomez-Bezares, F., \& Ugarte, J. V. (2015). Firm characteristics and intellectual capital disclosure in IPO prospectuses. Academia Revista Latinoamericana de Administración, 28(4), 461-483. https://doi.org/10.1108/arla09-2014-0134

An, Y., Davey, H., Eggleton, I. R., \& Wang, Z. (2015). Intellectual capital disclosure and the information gap: Evidence from China. Advances in Accounting, 31(2), 179-187. https://doi.org/10.1016/j.adiac.2015.09.001

April, K. A., Bosma, P., \& Deglon, D. A. (2003). IC measurement and reporting: Establishing a practice in SA mining. Journal of Intellectual Capital, 4(2), 165-180. https://doi.org/10.1108/14691930310472794

Babío, M. R., \& Muíño, M. F. (2005). Corporate Characteristics, Governance Rules and the Extent of Voluntary Disclosure in Spain. Advances in Accounting, 21, 299-331. https://doi.org/10.1016/s0882-6110(05)21013-1

Barako, D. G., \& Brown, A. M. (2008). Corporate social reporting and board representation: evidence from the Kenyan banking sector. Journal of Management and Governance, 12(4), 309-324. https://doi.org/10.1007/s10997-0089053-x

Barako, D. G., Hancock, P., \& Izan, H. Y. (2006). Relationship between corporate governance attributes and voluntary disclosures in annual reports: the Kenyan experience. Financial Reporting Regulation and Governance, 5(1), 1-27.

Beattie, V., \& Thomson, S. J. (2007). Lifting the lid on the use of content analysis to investigate intellectual capital 
disclosures. Accounting Forum, 31(2), 129-163. https://doi.org/10.1016/j.accfor.2007.02.001

Berle, A. A., \& Means, G. C. (1932). The Modern Corporation and Private Property. New York: Macmillan.

Bozzolan, S., Favotto, F., \& Ricceri, F. (2003). Italian annual intellectual capital disclosure: An empirical analysis. Journal of Intellectual Capital, 4(4), 543-558. https://doi.org/10.1108/14691930310504554

Brennan, N. (2001). Reporting intellectual capital in annual reports: Evidence from Ireland. Accounting, Auditing \& Accountability Journal, 14(4), 423-436. https://doi.org/10.1108/09513570110403443

Briano, G.C., \& Saavedra, M. L. (2015). The composition of the board and ownership structure as explanatory factors of transparency in corporate governance in Latin America: Evidence from listed companies in Argentina, Brazil, Chile and Mexico. Estudios Gerenciales, 31(136), 275-286. https://doi.org/10.1016/j.estger.2015.02.001

Briozzo, A., Albanese, D., \& Santolíquido, D. (2017). Gobierno corporativo, financiamiento y género: un estudio de las pymes emisoras de títulos en los mercados de valores argentinos. Contaduría y Administración, 62(2), 339-357. https://doi.org/10.1016/j.cya.2017.01.005

Brooking, A. (1997). El capital intelectual: el principal activo de las empresas del tercer milenio. Barcelona: Paidós Empresa.

Bueno Campos, E. (2002). El Capital Social en el nuevo enfoque del Capital intelectual de las organizaciones. Revista de Psicología del Trabajo y las Organizaciones, 18(2-3 ), 157-176.

Cerbioni, F., \& Parbonetti, A. (2007). Exploring the Effects of Corporate Governance on Intellectual Capital Disclosure: An Analysis of European Biotechnology Companies. European Accounting Review, 16(4), 791 - 826. https:// doi.org/10.1080/09638180701707011

Chen, C. J. P., \& Jaggi, B. (2000). Association between independent non-executive directors, family control and financial disclosures in Hong Kong. Journal of Accounting and Public Policy, 19(4-5), 285-310. https://doi.org/10.1016/ s0278-4254(00)00015-6

CNMV (1998). Informe del Comité Olivencia sobre el Gobierno de las Sociedades. Madrid: Consejo de Ministros

CNMV. (2003). Informe de la Comisión Especial para el fomento de la transparencia y seguridad en los mercados y en las sociedades cotizadas. Madrid: Consejo de Ministros.

CNMV (2006): Informe del grupo especial de trabajo sobre buen gobierno de las sociedades cotizadas. Madrid: Ministerio de Economía y Hacienda.

CNMV (2013): Código Unificado de Buen Gobierno de las empresas cotizadas. Madrid: Ministerio de Economía y Hacienda.

CNMV (2015): Unified code of corporate governance. Informe del grupo especial de trabajo sobre buen gobierno de las sociedades cotizadas. Madrid: Ministerio de Economía y Hacienda.

Coles, J. L., Daniel, N. D., \& Naveen, L. (2008). Boards: Does one size fit all? Journal of Financial Economics, 87(2), 329-356. https://doi.org/10.1016/j.jfineco.2006.08.008

de Cássia Fucci-Amato, R., \& Amato-Neto, J. (2010). A influência do capital humano e do capital intelectual no desenvolvimento de aglomerações de empresas e redes de cooperação produtiva. Journal of Technology Management \& Innovation, 3(2), 56-66.

De Silva, T. A., Stratford, M., \& Clark, M. (2014). Intellectual capital reporting: a longitudinal study of New Zealand companies. Journal of Intellectual Capital,15(1), 157-172. https://doi.org/10.1108/jic-03-2013-0034

Edvinsson, L., \& Malone, M. S. (1999). El capital intelectual: Cómo identificar y calcular el valor de los recursos intangibles de su empresa. Barcelona: Gestión 2000.

Eng, L. L., \& Mak, Y. T. (2003). Corporate governance and voluntary disclosure. Journal of Accounting and Public Policy, 22(4), 325-345. https://doi.org/10.1016/S0278-4254(03)00037-1

Gisbert, A., \& Navallas, B. (2013). The association between voluntary disclosure and corporate governance in the presence of severe agency conflicts. Advances in Accounting, 29(2), 286-298. https://doi.org/10.1016/j. adiac.2013.07.001

Goh, P. C., \& Lim, K. P. (2004). Disclosing intellectual capital in company annual reports: Evidence from Malaysia. Journal of Intellectual Capital, 5(3), 500-510. https://doi.org/10.1108/14691930410550426

Guthrie, J., \& Petty, R. (2000). Intellectual capital: Australian annual reporting practices. Journal of Intellectual Capital, 1(3), 241-251. https://doi.org/10.1108/14691930010350800

Guthrie, J., Petty, R., Yongvanich, K., \& Ricceri, F. (2004). Using content analysis as a research method to inquire into intellectual capital reporting. Journal of Intellectual Capital, 5(2), 282-293. https://doi. 
org/10.1108/14691930410533704

Hall, R. (1992). The strategic analysis of intangible resources. Strategic Management Journal, 13(2), pp. $135-144$. https://doi.org/10.1002/smj.4250130205

Haniffa, R. M., \& Cooke, T. E. (2005). The impact of culture and governance on corporate social reporting. Journal of Accounting and Public Policy, 24(5), 391-430. https://doi.org/10.1016/j.jaccpubpol.2005.06.001

Hernández, M., Aibar, B., \& Aibar, C. (2015). Determinants of corporate risk disclosure in large Spanish companies: a snapshot. Contaduría y Administración, 60(4), 757-775. https://doi.org/10.1016/j.cya.2015.05.014

Hernández, R., Fernández, C., \& Baptista, P. (2006). Metodología de la Investigación. México: McGraw-Hill Interamericana.

Herrera, E. E., \& Macagnan, C. B. (2016). Revelación de informaciones sobre capital estructural organizativo de los bancos en Brasil y España. Contaduría y administración, 61(1), 4-25. https://doi.org/10.1016/j.cya.2015.09.007

Hidalgo, R. L., \& García-Meca, E. (2009). Divulgación de información sobre el capital intelectual de empresas nacionales que cotizan en la Bolsa Mexicana de Valores. Contaduría y administración, (229), 105-131. http://dx.doi. org/10.22201/fca.24488410e.2009.658

Hidalgo, R. L., García-Meca, E., \& Martínez, I. (2011). Corporate governance and intellectual capital disclosure. Journal of Business Ethics, 100(3), 483-495. https://doi.org/10.1007/s10551-010-0692-X

Ho, S. S. M., \& Wong, K. S. (2001). A study of the relationship between corporate governance structures and the extent of voluntary disclosure. International Journal of Accounting, Auditing and Taxation, 10(2), 139-156. https://doi. org/10.1016/s1061-9518(01)00041-6

Jensen, M. C. (1993). The modern industrial revolution, exit, and the failure of internal control systems. Journal of Finance, 48(3), 831-880. http://dx.doi.org/10.1111/j.1540-6261.1993.tb04022.x

Jensen, M. C., \& Meckling, W. H. (1976). Theory of the firm: Managerial behavior, agency costs and ownership structure. Journal of Financial Economics, 3(4), 305-360. https://doi.org/10.1016/0304-405X(76)90026-X

Li, J., Pike, R., \& Haniffa, R. (2008). Intellectual capital disclosure and corporate governance structure in UK firms. Accounting and Business Research, 38(2), 137-159. https://doi.org/10.1080/00014788.2008.9663326

Lim, S., Matolcsy, Z., \& Chow, D. (2007). The Association between Board Composition and Different Types of Voluntary Disclosure. European Accounting Review, 16(3), 555 - 583. https://doi.org/10.1080/09638180701507155

Meritum (2002). Guidelines for managing and reporting on intangibles. Fundación Airtel-Vodafone.

OECD, Organisation for Economic Co-operation and Development. (2001). OECD Science, Technology and Industry Scoreboard 2001: Towards a Knowledge-based Economy. Paris: OECD Publishing.

Oliveras, E., \& Kasperskaya, Y. (2005). Reporting Intellectual Capital in Spain Economics and Business Working Papers Series 781. Barcelona: Universidad Pompeu Fabra.

Pearce, J. A., \& Zahra, S. A. (1992). Board compensation from a strategic contingency perspective. Journal of Management Studies(29), 411-438.

Prado, J. M., García, I. M. \& Gallego-Álvarez, I. (2009). Características del consejo de administración e información en materia de responsabilidad social corporativa. Revista Española de Financiación y Contabilidad, 38(141), 107135. https://doi.org/10.1080/02102412.2009.10779664

Rodrigues, L. L., Tejedo-Romero, F., \& Craig, R. (2016). Corporate governance and intellectual capital reporting in a period of financial crisis: Evidence from Portugal. International Journal of Disclosure and Governance. https:// doi.org/10.1057/jdg.2015.20

Rodríguez-Ariza, L., Frías, J. V. \& García, R. (2014). El consejo de administración y las memorias de sostenibilidad. Revista de Contabilidad, 17(1), 5-16. https://doi.org/10.1016/j.rcsar.2013.02.002

Sánchez Fernández de Valderrama, J. L. (2004). Información corporativa, opciones contables y análisis financiero: Publicaciones de la Real Academia de las Ciencias Económicas y Financieras.

Sonnier, B.M. (2008). Intellectual capital disclosure: high-tech versus traditional sector companies. Journal of Intellectual Capital, 9(4), 705-722. https://doi.org/10.1108/14691930810913230

Spagnol, R., Moraes, R. D. O., \& Piqueira, J. R. C. (2015). Knowledge Management as a Competitive Advantage to the Brazilian MVAS Ecosystem.Journal of Technology Management \& Innovation, 10(2), 1-8. http://dx.doi. org/10.4067/S0718-27242015000200001

Sveiby, K. E. (1997). The intangible assets monitor. Journal of Human Resource Costing and Accounting, 2(1), 73-97. 
https://doi.org/10.1108/eb029036

Tabares, A., Alvarez, C., \& Urbano, D. (2015). Born Globals From the Resource-Based Theory: a Case Study in Colombia. Journal of Technology Management \& Innovation, 10(2), 155-165. https://doi.org/10.4067/s071827242015000200011

Tejedo-Romero, F. \& Araujo, J. F. (2015). A gestão da informação do conhecimento organizacional das empresas. Observatorio $\left(O B S^{*}\right), 9(2), 189-205$.

Vandemaele, S. N., Vergauwen, P. G. M. C., \& Smits, A. J. (2005). Intellectual capital disclosure in The Netherlands, Sweden and the UK: A longitudinal and comparative study. Journal of Intellectual Capital, 6(3), 417-426. https:// doi.org/10.1108/14691930510611148

Villegas González, E., Hernández Calzada, M. A., \& Salazar Hernández, B. C. (2017). La medición del capital intelectual y su impacto en el rendimiento financiero en empresas del sector industrial en México. Contaduría y Administración, 62(1), 184-206. https://doi.org/10.1016/j.cya.2016.10.002

Wagiciengo, M. M., \& Belal, A. R. (2012). Intellectual capital disclosures by South African companies: A longitudinal investigation. Advances in Accounting, 28(1), 111-119. https://doi.org/10.1016/j.adiac.2012.03.004

Whittington, G. (1993). Corporate Governance and the Regulation of Financial Reporting Accounting and Business Research, 23(91A), 311-319. https://doi.org/10.1080/00014788.1993.9729899

Zerenler, M., Hasiloglu, S. B., \& Sezgin, M. (2008). Intellectual capital and innovation performance: empirical evidence in the Turkish automotive supplier. Journal of Technology Management \& Innovation, 3(4), 31-40. https://doi. org/10.4067/s0718-27242008000200003 